\title{
A natural and biodegradable scaffold of electrospun eggshell membrane
}

\author{
W. D. Kim, T. Min, S. A. Park, J. H. Park \& G. H. Kim \\ Bio-mechatronic Division, Department of Future Technology, \\ Korea Institute of Machinery and Materials (KIMM), Korea
}

\begin{abstract}
A successful tissue-engineering scaffold must have a highly porous structure and good mechanical stability. High porosity and optimally designed pore size provide structural space for cell accommodation and migration and enable the exchange of nutrients between the scaffold and cell culturing environment. The pore structures should be interconnected with each other for cell growth and proliferation. Soluble eggshell membrane protein (SEP) was prepared from eggshell membrane (ESM) and mixed with poly( $\varepsilon$-caprolactone) (PCL) solutions. SEP/PCL micro-size-fibre web was fabricated using electrospinning process with the help of a dual nozzle and auxiliary cylindrical electrode. The web was characterized with water contact angle (WCA) and cellular behaviour. The SEP/PCL web, which showed feasibility to fabricate scaffold having adequate hydrophilicity and suitable pore distribution, was a good example mimicking the natural biomaterial.
\end{abstract}

Keywords: biomimic, soluble eggshell protein, poly(e-caprolactone), nanofibre.

\section{Introduction}

Natures generate remarkable materials such as abalone nacre, sea mussel adhesive, and spider silk, which is an extremely strong material and is on weight basis stronger than steel. Biomimic is a science, which takes inspiration from Natures' designs and processes to solve engineering problems [1]. Scientists and engineers are reverse engineering many of animals' performance characteristics using these advances. These research areas include materials, actuators, sensors, structures, functionality, control, intelligence and autonomy [2]. Recently, these technological trends using the nature-inspired technology were expanded in the 
field of biological areas. Realistic achievements have been obtained at the interface of nano-materials and biology, including the fabrication of nanofibre materials for three-dimensional scaffold and tissue engineering, the creation of living micro-lenses, and the synthesis of metal nano-wires on DNA templates [3].

As stated by previous researchers $[4,5]$, the scaffold consisting of nanofibres overcomes the limitations of conventionally fabricated scaffolds due to their high surface area and high porosity. According to Buckley and O'Kelly [6], ideal scaffolds should possess the following characteristics to bring about the desired biological response: (1) highly porous with an interconnected pore network and flow transport of nutrients and metabolic waste, (2) biodegradable with a controllable degradation, (3) suitable surface chemistry for cell attachment, (4) mechanical properties, and (5) be easily processed to form a variety of shapes and sizes.

Considering the previously stated technical points of ideal scaffolds, eggshell membrane (ESM) has interesting structures and functions, such as interconnected porous structure, good hydrophilic surface property, and transporting function of nutrients to the chick's embryo.

The ESM was composed with a double-layered structure in which outer structure is randomly entangled micro-size fibres with the average diameter of 3 $\mu \mathrm{m}$ (figure 1). Despite of various researches for the applications using the biological composition of the ESM, few applications were reported for the biomimical regeneration of the unique structure of the membrane.

We used in this paper poly(e-caprolactone) (PCL), which presents good mechanical properties relative to other biomaterials and has been developed as a biomaterial scaffold having biodegradability and bioreabsorption properties [7].

In this work, we imitate the natural micro-fibrous structure of the eggshell membrane as a biomedical scaffold. In order to obtain this goal, firstly we analyse the structure of eggshell membrane, such as pore size, porosity, and water contact angle (WCA). The soluble eggshell protein (SEP) was extracted from the eggshell membrane, and it was used to fabricate micro-fibre web using electrospinning process. To increase the processability of the process, SEP was co-extruded with a biocompatible PCL. The mixture was electrospun using a dual nozzle and auxiliary electrode to achieve stable initial jets at a nozzle tip. The fabricated bio-composite was characterized in WCA and cellular behaviour. The growth characteristic of human dermal fibroblasts (HDFs) cultured on the spun mat showed the good attachment relative to pure PCL webs.

\section{Experimental method}

\subsection{Materials}

The extraction of SEP from ESM was followed by the experimental method of Yi et al. [8]. The protein was acquired dissolving raw ESM in aqueous 3-mecaptopropionic acid and $10 \%$ of acetic acid, and the solution was heated to $85^{\circ} \mathrm{C}$ for $12 \mathrm{~h}$. After cooling the traces of insoluble solid in the solution was removed 
by centrifuging. The solution was adjusted to $\mathrm{pH} 5$ with $5 \mathrm{~mol} / \mathrm{L}$ of $\mathrm{NaOH}$. The white precipitate was acquired by filtration and washed with ethanol. $2 \mathrm{wt} \%$ of SEP solutions were prepared by dissolving $0.2 \%$ of aqueous $\mathrm{NaOH}$ with prepared SEP. Yi et al. [8] used poly(ethylene oxide) (PEO) to increase the spin-ability of this process. However, SEP/PEO solution was difficult to accumulate spun fibre on a dielectric surface due to the surface charges of the electrospun fibres. Moreover, the PEO cannot conduct a role as a matrix of a SEP/PEO web because of its great solubility to water during cell culturing. For that reason, we used poly( $\varepsilon$-caprolactone) (PCL, Sigma-Aldrich) which could be used for increasing spin-ability. $8 \mathrm{wt} \%$ of PCL was dissolved in a solvent mixture of methylene chloride (MC)/dimethyl formamide (DMF) at a ratio of 20/80. The each solution was injected into the dual nozzles with a $20 \mathrm{~mL}$ glass syringe. The flow rates of the solutions were controlled using two syringe pumps (KDS 230; NanoNc Inc., Seoul, Korea).

(a) inside surface

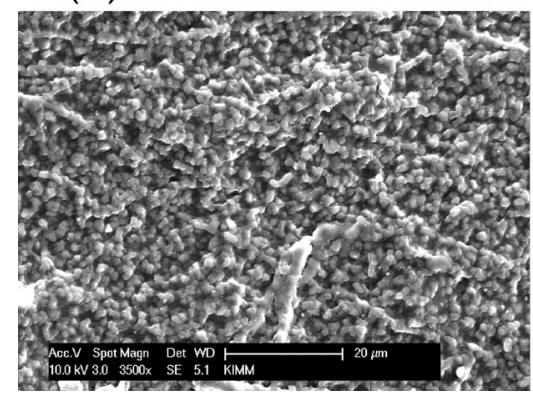

(b) outside surface

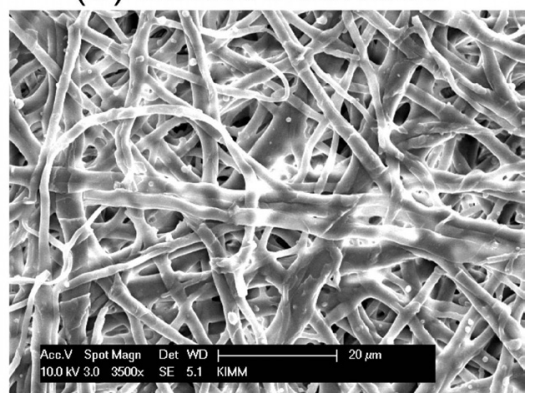

Figure 1: SEM images of ESM, (a) for inside surface structure and (b) outside surface of the membrane.

\subsection{Electrospinning}

Micro/nanofibres could have been fabricated using an electrospinning technique. The principle of electrospinning is that a Taylor cone is formed by applying an electric field to polymer solution hanging from a capillary tip, which causes jets of electrically charged solution to be emitted when the applied electrostatic force is stronger than the surface tension of the solution. The jets of solution erupt from the apex of the cone at a nozzle and travel toward an electrically grounded target on which they are stacked as a nonwoven mat.

The SEP/PCL nanofibres were fabricated using an electrospinning technique with a cylindrical auxiliary electrode and dual nozzle system. The cylindrical electrode and nozzle were connected by a copper wire and could be charged simultaneously as the fluid passed through the spinneret. In the dual nozzle, the two different solutions were injected into the capillaries of the nozzle. Outer part $\left(\mathrm{S}_{1}\right)$ was fed with the PCL and inner nozzle $\left(\mathrm{S}_{2}\right)$ with SEP. The feeding rates for the solutions of SEP and PCL were 0.25 and $0.5 \mathrm{mLh}^{-1}$, respectively. The poly(ethylene terephthalate) (PET) film covered a grounded electrode as a 
collector. The detailed shape and geometry of the auxiliary electrode and dual nozzle are shown in figure 2. The cylindrical electrode served to reduce the instability of the initial jet leaving the apex of the Taylor cone. More details on the role of the electrode are described below.

The syringe was subjected to the same applied voltage $(19.2 \mathrm{kV})$ with a distance of $100 \mathrm{~mm}$ between the target and needle tip, and a high-voltage power supply (SHV300RD-50K; Convertech, Korea) was used to control the applied voltage. The initially spun jet was photographed using a digital camera (E-300; Olympus, Tokyo, Japan). The spun fibres were deposited on a PET film covering a copper target.

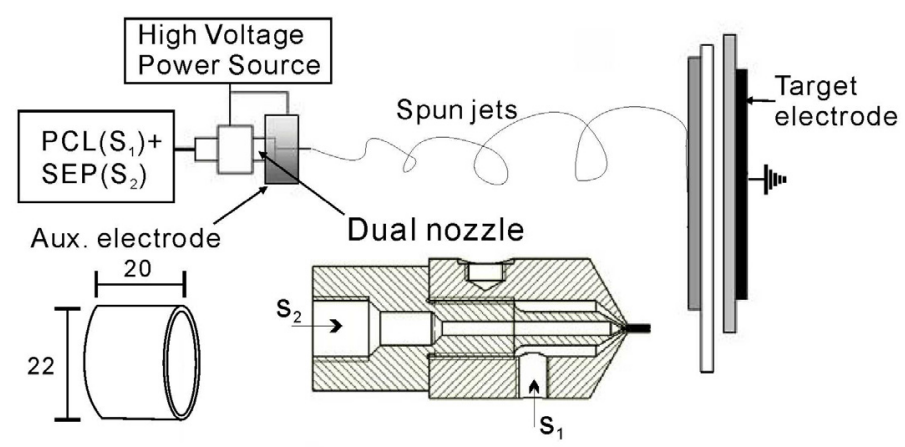

Figure 2: Schematic of the electrospinning setup with an auxiliary cylindrical electrode and dual nozzle. The cylindrical electrode has a radius of $11 \mathrm{~mm}$ and is $0.4 \mathrm{~mm}$ thick.

\subsection{Cell culture and experiment}

Primary fibroblasts were isolated from sterile biopsies of normal skin. The specimens were minced in tissue culture dishes and cultured in an atmosphere of $5 \% \mathrm{CO}_{2}$ and $37^{\circ} \mathrm{C}$. The nanofibre scaffolds were sterilized with $70 \%$ ethanol and exposed to UV light for 1 hour to eliminate contamination. And, they were prewarmed with Hank's balanced salt solution (HBSS) for 4 hours. Human dermal fibroblasts (HDFs) were cultured in Dulbecco's Modified Eagle Medium (DMEM) supplemented with $10 \%$ fetal bovine serum (FBS) and $1 \%$ antibiotics (penicillin/streptomycin). The cells were maintained up to passage 7 . Isolated fibroblasts were seeded on each sample $(1 \times 1 \mathrm{~cm})$ at a density of $5.0 \times 10^{4}$ cells/nanofibre and cultured for up to 3 days. The cells were fixed with $4 \%$ glutaraldehyde for 1 hour at room temperature, and dehydrated through a series of ethanol dilutions. The samples were sputter-coated with Pt. Cell morphology and growth was observed using a scanning electron microscopy (FE-SEM, Sirion; FEI, Hillsboro, OR, USA).

\subsection{Structural morphology}

The morphology of the electrospun mat of the mixture (SEP/PCL) was observed using an optical microscope (BX FM-32; Olympus) and SEM. Before 
observation, the scaffolds were coated with gold using a sputter coater. The pore size, total pore volume, pore area, and porosity were characterized with the AutoPore III mercury porosimeter (Micromeritics Instrument Co., Norcross, GA). Sample preparation and procedures for measurement were conducted following the instructions provided by the manufacturer.

\section{Results and discussion}

\subsection{The characterization of ESM}

It has been well known that surface roughness and chemical structure of polymeric scaffolds are key roles on cell growth and proliferation [9]. Figure 3 shows the WCA of ESM. The comparison of the measured contact angle between the inner and outer surface of eggshell membrane, which was shown in figure 1, shows dramatically different result. The outer surface of ESM shows good hydrophilic property, but hydrophobic for the inner surface. Since the specific surface property of the membrane, the eggshell membrane may have been researched for a selective filtering system [10].

(a) inner surface

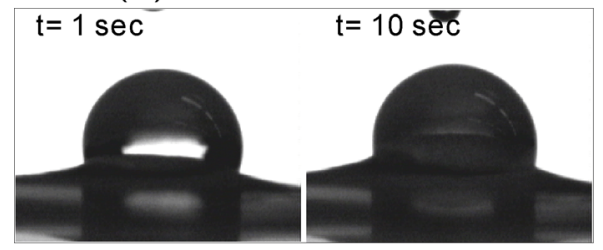

(b) outer surface

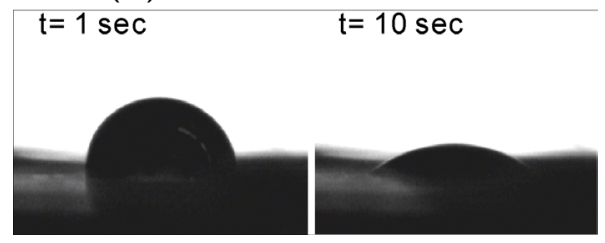

Figure 3: Photographs taken during WCA measurements: (a) inner surface of $\operatorname{ESM}\left(\mathrm{WCA}=95^{\circ}\right.$ at $\mathrm{t}=1 \mathrm{sec}, 93^{\circ}$ at $\left.\mathrm{t}=10 \mathrm{sec}\right)$, (b) outer surface of $\operatorname{ESM}\left(\mathrm{WCA}=80^{\circ}\right.$ at $\mathrm{t}=1 \mathrm{sec}, 33^{\circ}$ at $\left.\mathrm{t}=10 \mathrm{sec}\right)$.

In figure 4, assessment of structural pore property of ESM was determined with the use of a mercury porosimeter. The porosity of the ESM was $59.62 \%$. Total pore volume was $2.09 \mathrm{~mL} / \mathrm{g}$ and total pore size was $232.96 \mathrm{~m}^{2} / \mathrm{g}$. The plot against pore size diameter indicated that the pore size had broad range from 0.005 to $500 \mu \mathrm{m}$.

As shown in the figure 1, ESM has consisted of two layers and the composition of the membrane can be separated into two parts, insoluble and soluble. According to the pre-treatment of ESM, the yield of the soluble protein, 
SEP, was less than $63 \%$ and it means the insoluble part can be exist in some structure. Figure 5 shows an image of insoluble remnant after $24 \mathrm{~h}$ of chemical treatment. The transmission microscope images show the diameter of the ESM fibres was dramatically decreased after removing the soluble protein. From the result, we can find that the soluble and insoluble part is coexisted with fibrous forms.

In summary of the analysis of ESM, the detail structure and surface properties are described in Table 1.

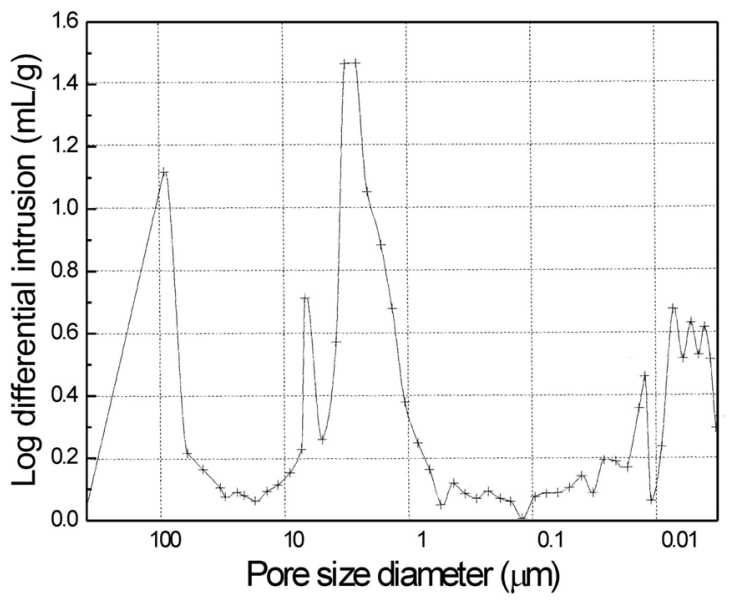

Figure 4: Plot against pore size diameter of ESM. The differential intrusion means the relative quantity of pores of a specific diameter.

(a) before treatment

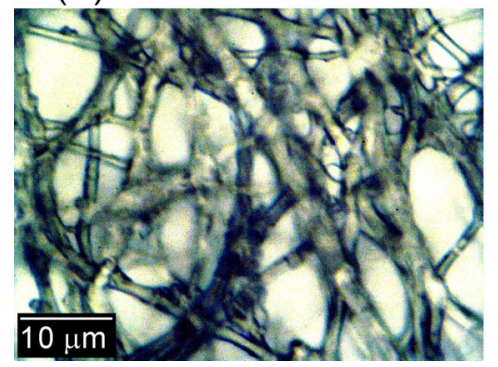

(b) after treatment

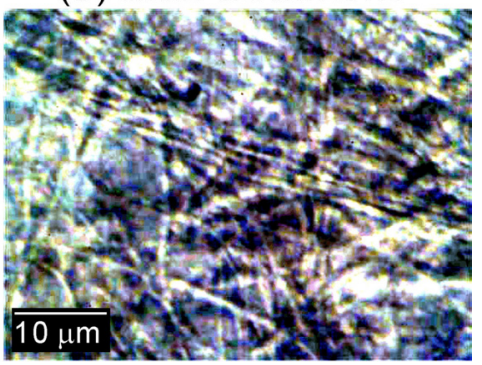

Figure 5: The images taken by the transmission optical microscope. (a) is pure ESM and (b) is insoluble remnant of ESM which was chemical treated.

\subsection{Regenerations of structure of ESM}

To mimic the topography of the outer eggshell membrane, we fabricated SEP/PCL nanofibres using a specially designed electrospinning process, in 
which a dual nozzle is attached to the syringe pumping system and a supplementary cylindrical electrode. When various electric-field conditions and processing conditions enforced to the solution, the size-controllable micro/nanosized fibres were obtained (figure 6). Figures 6(a) and (b) show the contours of the electric field with the same electric field scale near the nozzle for a standard electrospinning and the process supplemented using a cylindrical electrode. The distribution of the electric field inferred from the shape of the auxiliary electrode was analysed using the commercial software ANSYS/Emag for the electromagnetic analysis. The numerical models consisted of two-dimensional Plane121 elements and 8-Node charge-based electric elements. From the calculated and experimental results, the auxiliary electrode altered the distribution of the electric field near the nozzle tip. In the normal nozzle without the auxiliary electrode, the direction of the initial spun solution at the nozzle tip was instable, due to near environmental conditions, figure 6(a). In contrast, with the supplementary electrode, the initial direction of the solution was very stable and the jet toward the counter electrode was straight, figure 6(b).

Table 1: $\quad$ Properties of ESM.

\begin{tabular}{lcc}
\hline & \multicolumn{2}{c}{ Eggshell membrane } \\
\cline { 2 - 3 } Structure & inner surface & outer surface \\
diameter (average) & $2 \mu \mathrm{m}$ (bead) & $3 \mu \mathrm{m}$ (fibre) \\
pore size (total) & \multicolumn{2}{c}{$232.96 \mathrm{~m}^{2} / \mathrm{g}$} \\
pore volume & $2.09 \mathrm{~mL} / \mathrm{g}$ \\
porosity & $59.62 \%$ & \\
WCA $^{*}$ & \multicolumn{3}{c}{$93^{\circ}$} \\
$\mathrm{t}=1 \mathrm{~s}$ & $95^{\circ}$ & $33^{\circ}$ \\
$\mathrm{t}=10 \mathrm{~s}$ & $80^{\circ}$ & \\
\hline *: Water contact angle. & &
\end{tabular}

Figure 7 shows transmission optical microscope images of the electrospun PCL and SEP/PCL fibres deposited on the PET film. The electrospun webs were consisted of randomly distributed non-woven fibres and the diameters of the PCL and the mixture are similar each other, showing an average diameter $2.3 \mu \mathrm{m}$.

To check the increase of hydrophilic property of the mixture (SEP/PCL), the measurements of the WCA were performed. Figure 8 shows the contact angles of the samples electrospun with and without SEP solution. It is clear that the spun web coextruded with the eggshell protein solution and PCL solution leads to a lower contact angle, i.e. higher hydrophilic property, which may be one of the reasons for enhancing the attachment of the seeding cells. According the results, SEP injected to the inner nozzle was mixed with PCL rather than forming a core- 
shell structure. It is because the SEP solution may disperse during the spinning process, so that the spun jets appear to be not coated with PCL solutions, even though SEP was injected inside of nozzle. Resultantly, we can conclude that the nanofibre web of the mixture has hydrophilic property relative to that of pure PCL nanofibre web.

(a) w/o aux. electrode
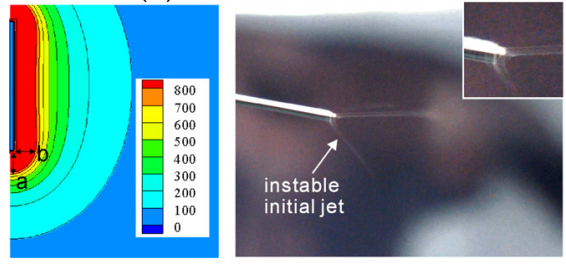

(b) with aux. electrode
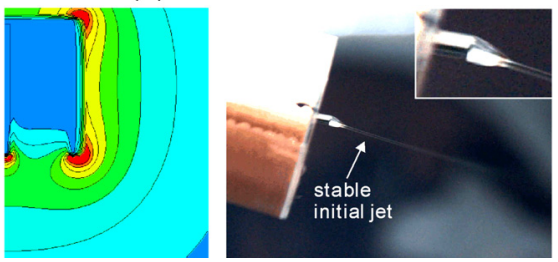

Figure 6: (a) and (b) are the contours of the electrical fields using without and with an auxiliary electrode and photographs of the Taylor cone at the nozzle tip: (a) normal electrospinning and (b) cylindrical auxiliary electrodes under the same applied electric voltage of $15 \mathrm{kV}$.

(a) PCL

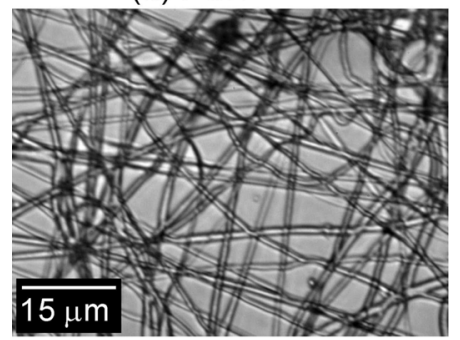

(b) SEP/PCL

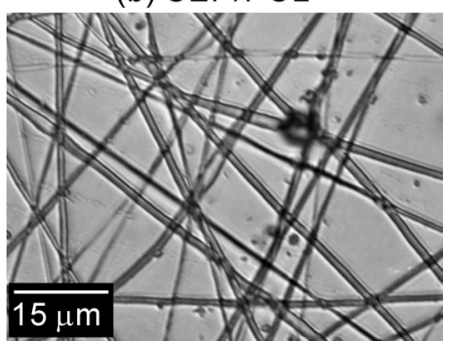

Figure 7: The images show the electrospun fibres of (a) PCL and (b) SEP/PCL: an average diameter of the fibres is $2.3 \mu \mathrm{m}$.

\subsection{Cell culturing}

The surface property of the biomaterial can control cell adhesion, shape, proliferation and function. Figure 9 shows SEM observation of HDFs on the PCL and SEP/PCL nanofibre web. Cell morphology in the SEM images had a multiple cell attachments on the webs. At 3 days, large area of confluence, which 
was tightly packed with the cells, was observed on the SEP/PCL web relative to the PCL web. As the cultured images are compared in viewpoint of the cell attachment of HDFs, the SEP/PCL web provides more uniformly distributed cells, while those were not in the PCL web.

The result of cell culture can be elucidated by the contact angle results. As shown in the figure 8, the electrospun SEP/PCL web had a lower WCA than that of PCL web and this phenomenon is effective in the cell attachment. It is because the hydrophilic SEP is appropriately well mixed with the PCL through the electrospinning process and the nanofibrous web-structure provides a high level of surface area for the cell attachment. Also, improved protein adsorption can enhance cell attachment to the substrate.

(a) PCL web

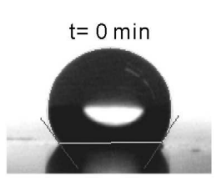

(b) SEP/PCL web

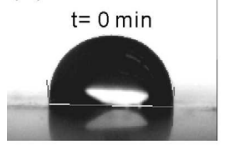

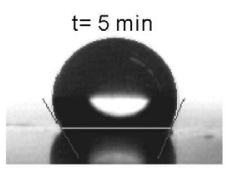

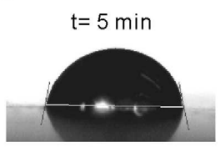

(c) water contact angle

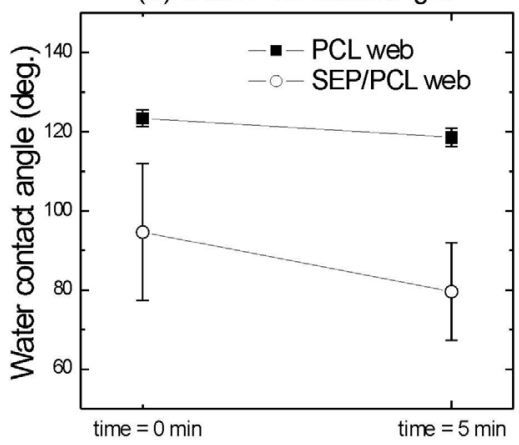

Figure 8: WCAs for (a) PCL and (b) SEP/PCL nanofibre web and (c) a compared plot against time.

(a) PCL web

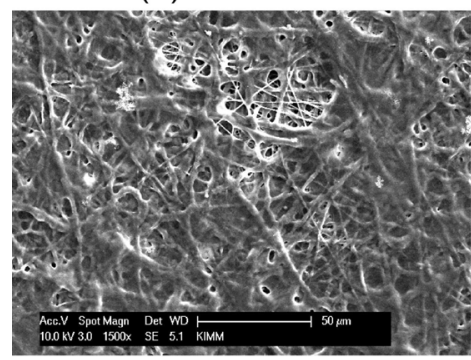

(b) SEP/PCL web

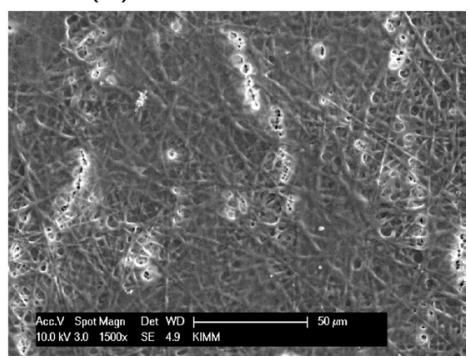

Figure 9: $\quad$ SEM images of the cultured HDFs on (a) PCL and (b) SEP/PCL nanofibre webs at 3 days.

\section{Conclusions}

In this paper, we tried to mimic the eggshell membrane, which has the characteristics of a biomedical scaffold such as interconnected porous structure, good hydrophilic surface property, and transportation system of nutrients. 
Although the ESM has various advantages as a scaffold, it has been difficult to change to adaptable shapes like an example of "tailor-made" scaffolds. To achieve the shape-ability of the ESM, we used electrospinning process to be able to generate various size fibres and shapes. To increase processability of SEP and conduct a role as the insoluble matrix of ESM, the biocompatible PCL was co-extruded. The solutions were electrospun using a dual nozzle and auxiliary cylindrical electrode. The SEP/PCL was easily fabricated into a nanofibrous web consisted of uniform sized fibres, and the fibrous web showed a good hydrophilic property like the ESM. From cell culturing experiments, the web enhanced the cell attachment relative to pure PCL nanofibre web, as observed in SEM images. In this paper, we only used HDFs for cell culture. Since cell attachment and cellular behavior within scaffold are generally dependent on cell type, we need to further studies dealing with the cell attachment using various cell types to confirm the feasibility of ESM as cell culturing.

\section{References}

[1] Benyus, J. M. Biomimicry, Innovation Inspired by Nature. William Morrow and Company. New York 1997.

[2] Bar-Cohen, Y \& Breazeal, C. Biologically Inspired Intelligent Robotics. Proceedings of the SPIE Smart Structures Conference, 5051-02, 2003.

[3] Zhang, S. Emerging biological materials through molecular self-assembly, Biotechnology Advances 20, 321-339, 2002.

[4] Li, W-J, Laurencin, C.T., Caterson, E.J., Tuan, R.S. \& Ko, F.K. Electrospun nanofibrous structure: A novel scaffold for tissue engineering, J Biomed Mater Res 60, 613-621, 2002.

[5] Zong, X., Bien, H., Chung, C-Y., Yin, L., Fang. D., Hsiao, B.S., Chu, B. \& Entcheva, E. Electrospun fine-textured scaffolds for heart tissue constructs. Biomaterials 26, 5330-5338, 2005.

[6] Buckley, C.J \& O'Kelly, K.U. Regular Scaffold Fabrication Technique for Investigations in Tissue Engineering (Prendergast, P.J and McHugh P.E. Eds.), Topics in Bio-Mechanical Engineering, 147-166, 2004.

[7] Pitt CG. Poly-epsilon-caprolactone and its copolymers. In: Chasin M, Langer, R, editors. Biodegradable polymers as drug delivery systems. New York: Marcel Dekker; 1990. p71-120.

[8] Yi, F., Yu, J., Guo, Z., Zhang, L. \& Li, Q. Natural Bioactive Material: A Preparation of Soluble Eggshell Membrane Protein, Macromol. Biosci. 3, 234237, 2003.

[9] Kaufmann, P.M., Heimrath, S., Kim, B.S. \& Mooney, D.J. Highly porous polymer matrices as a three-dimensional culture system for hepatocytes. Cell Transplant, 6, 463-468, 1997.

[10] Ishikawa, S., Suyama, K., Arihara, K., \& Itoh, M., Uptake and recovery of gold ions from electroplating wastes using eggshell membrane. Bioresources Technology 81, 201-206, 2002. 